\title{
Origin and fate of pseudogenes in Hemiascomycetes: a comparative analysis
}

\author{
Ingrid Lafontaine ${ }^{* 1,2}$ and Bernard Dujon 1,2,3
}

\begin{abstract}
Background: Pseudogenes are ubiquitous genetic elements that derive from functional genes after mutational inactivation. Characterization of pseudogenes is important to understand genome dynamics and evolution, and its significance increases when several genomes of related organisms can be compared. Among yeasts, only the genome of the $S$. cerevisiae reference strain has been analyzed so far for pseudogenes.
\end{abstract}

Results: We present here the first comparative analysis of pseudogenes within the fully sequenced and annotated genomes of eight yeast species, spanning the entire phylogenetic range of Hemiascomycetes. A total of 871 pseudogenes were found, out of which mutational degradation patterns and consequences on the genetic repertoire of each species could be identified. We found that most pseudogenes in yeasts originate from mutational degradation of gene copies formed after species-specific duplications but duplications of pseudogenes themselves are also encountered. In all yeasts, except in Y. lipolytica, pseudogenes tend to cluster in subtelomeric regions where they can outnumber the number of functional genes from 3 to 16 times. Pseudogenes are generally not conserved between the yeast species studied (except in two cases), consistent with their large evolutionary distances, but tend to be conserved among S. cerevisiae strains. Reiterated pseudogenization of some genes is often observed in different lineages and may affect functions essential in S. cerevisiae, which are, therefore, lost in other species. Although a variety of functions are affected by pseudogenization, there is a bias towards functions involved in the adaptation of the yeasts to their environment, and towards genes of unknown functions.

Conclusions: Our work illustrates for the first time the formation of pseudogenes in different branches of hemiascomycetous yeasts, showing their limited conservation and how they testify for the adaptation of the yeasts functional repertoires.

\section{Background}

Since their original discovery [1], pseudogenes have been found in all studied genomes so far, within the three kingdoms of life [2-4]. Their proportions vary greatly, however, from one organism to another, depending on lifestyle (free-living or association) and on genome properties (rates of duplication, mutation, deletion, and retrotransposition). Pseudogenes correspond to $c a$. $3 \%$ of the gene repertoire of Drosophila melanogaster, while there are approximately as many pseudogenes as functional genes in the human genome (http://pseudogenes.org). Pseudogenes are often species-specific and, within small genomes, tend to accumulate in chromosomal regions

\footnotetext{
* Correspondence: ingrid.lafontaine@pasteur.fr

1 Unité de Génétique Moléculaire des Levures, Institut Pasteur, Paris, France Full list of author information is available at the end of the article
}

such as subtelomeres or heterochromatin, minimizing possible deleterious effects [5-7].

The historical definition of a pseudogene is a DNA sequence that looks like an active gene but has lost its ability to code for a functional product, due to more or less extensive mutational disablements. Pseudogene formation is frequently observed in pathogenic organisms undergoing reductive evolution while benefiting from host functions [8-11]. Pseudogenes can also correspond to the non-functionalization of a duplicated gene copy $[12,13]$ that originated either from DNA duplication or from retro-transposition [14,15]. Sequence degradation may range from a single disabling mutation, such as a frameshift or an in-frame stop codon in a protein-coding gene, to extensive changes including numerous insertions and deletions $[7,16]$. 
In all genomes, most pseudogenes are likely to disappear with time by the accumulation of successive mutations [9]. Those with limited alterations, however, may be repaired by reverse mutations, gene conversion or may be reactivated by translational recoding events $[17,18]$. Some pseudogenes can also acquire a new functional role, such as the control of another gene expression, or the generation of genetic diversity (see [19] for a review), and give rise to new genes, such as the human XIST noncoding RNA gene, which evolved from a pseudogene of a protein-coding sequence [20].

In Fungi, only pseudogenes of particular genes or pathways of interest have been described [21-24]. In Saccharomyces cerevisiae, two systematic analyses performed by different approaches $[6,7]$ both concluded to the paucity of pseudogenes of anciently protein-coding sequences in the yeast genome. In order to determine the mechanisms of pseudogene formation across several related species, their age and the functions affected by pseudogenization, we performed the first systematic comparative analysis of the pseudogene repertoire in a set of eight hemiascomycetous yeasts spanning a large evolutionary range, similar or larger than the phylum of Chordates [25,26]. We show that these genomes also contain a limited number of pseudogenes, independently of their global level of gene redundancy. Most pseudogenes originate from duplicated gene copies resulting from previous DNA duplication events, but a few could correspond to retroprocessed sequences. Some pseudogenes are formed from single-copy gene and hence correspond to a functional loss in the yeast species. The relative paucity of pseudogenes suggests that functionally inactive genes are rapidly eliminated in hemiascomycetous genomes because their sequence diverges rapidly or because they are successively truncated, or entirely deleted.

\section{Methods}

\section{Genomes and protein sequences}

Sequences and annotations were taken from the Génolevures database (http://www.genolevures.org) for Candida glabrata, Zygosaccharomyces rouxii, Kluyveromyces lactis, Kluyveromyces thermotolerans, Saccharomyces kluyveri, Debaryomyces hansenii and Yarrowia lipolytica, and from the Saccharomyces Genome Database (http:// www.yeastgenome.org/) for Saccharomyces cerevisiae. All these sequenced strains are haploids. Note that the annotation files already contained 457 protein-coding pseudogenes. The protein databank used for comparison contains the translational products of the 44,174 annotated CDS from all eight studied genomes (http:// www.genolevures.org/yeastgenomes.html). We used the protein family classification constructed by the Génolevures consortium [27].
Whole genome shotgun sequences of $S$. cerevisiae haploid strains AWRI1631, JAY291, M22, RM11-1a, YJM789, YPS163 and EC1118 were retrieved at EMBL (EMBL: ABSV00000000, ACFL $01000000, \quad$ ABPC 00000000 , AAEG01000000, AAFW00000000 and ABPD00000000, respectively), as well as the genomic scaffolds of the strain EC1118 (EMBL: FN393058-FN393060, FN393062FN393087, FN394216, and FN394217).

\section{Identification of the pseudogenes}

We developed a set of automatic procedures to obtain an exhaustive list of the potential pseudogenes of anciently protein-coding genes in a given genome. Each genome sequence is given to FASTY [28] and translated into the 6 frames for detection of amino acid similarity against the translational products of the 44,174 annotated CDS from the studied genomes (no pseudogenes from full protein sequences encoded in the nuclear genomes could be retrieved outside of the hemiascomycetes in the Uniprot database).

The matches selected have at least $25 \%$ identity and are considered statistically significant if their estimated Zscore [29] is greater or equal to 200 (corresponding to Evalues lower than $10^{-6}$ ). This cut-off was chosen after examining the distribution of the Z-scores for all obtained results. When several matches overlap, the similarity region is delimited from the left-most to the rightmost aligned sequences.

\section{Protein domain conservation}

All matches similar to a protein of unknown function were queried against the PFAM database [30] of conserved domains with the HMMER algorithm[31].

\section{Identification of the pseudogenes in S. cerevisiae strains}

The regions corresponding to the pseudogenes in the $S$. cerevisiae reference strain S288C were retrieved in the whole genome shotgun sequences of the strains AWRI1631, EC1118, JAY291, M22, RM11-1a, YJM789 and YPS163 by a FASTA search at the nucleotide level. These identified regions were then queried against the protein bestmatches of the corresponding pseudogenes in S288C with FASTY. We concluded to the presence of a pseudogene in the considered strain if evidence for coding sequence degradation is proposed in the FASTY alignment (see criteria 3 in section below). The pseudogenes present in strains M22 and YPS163 but not in S288C were identified based on the work by [32].

\section{Selection criteria}

Pseudogenes are defined from the similarity regions using the following criteria: 1) no overlap or partial overlap with an already annotated functional genetic element on the same DNA strand; 2) possible overlap of less than 100 nucleotides with an already annotated functional 
genetic element on the opposite DNA strand; 3) evidence for coding sequence degradation: at least one in-frame stop codon, one frameshift mutation, or a truncation of at least $30 \%$ relatively to the cognate CDS (truncated pseudogenes smaller than 120 nucleotides are ignored, unless their cognate CDS is itself smaller than 120 nucleotides).

Each selected potential pseudogene is aligned to its bestmatch with GENEWISE [33] to predict the corresponding coding sequence. Fifteen candidates were discarded after this step because GENEWISE predicted an intact CDS while FASTY introduced a frameshift mutation in the alignment.

The pseudogenes identified in this study are available at the Génolevures website (http://www.genolevures.org/). The algorithm for pseudogene detection was written for our own analysis, considering that annotations of the genomes studied are of high quality. It is available upon request to the authors.

\section{Pseudogene nomenclature}

Each detected pseudogene is numbered serially from left to right of the chromosome, based on the nomenclature proposed in [34]. The name indicates the species (four letters), the project or strain number (one numeral), the chromosome (one letter), and the pseudogene nature of the sequence ("p") followed by the serial number (for example, CAGLOAp1).

\section{Sequence divergence analysis}

We assumed that substitutions at the third codon position of a protein coding sequence (or an ancient protein coding sequence) evolve according to a neutral molecular clock. Estimations of the evolutionary distances by several substitution models (JC69, F84 and HKY85) are saturated for half of our data set and, therefore, were not retained for analysis. P-distances are then computed for amino acid and for nucleotide sequences on the third codon position. To conserve the frame of the coding sequences, the nucleotide alignment is derived from the amino acid alignment (in-house script) of the translated products of the pseudogene and its cognate gene obtained by GENEWISE. The nucleotide alignments between functional paralogs are derived from the amino acid alignments obtained by MUSCLE [35].

\section{Synteny conservation}

Pairwise synteny was examined in windows of five CDS upstream and five CDS downstream of the pseudogene and any of its homolog in another genome. We confirmed conservation of synteny if at least two pairs of homologous neighbors are found within the window.

\section{Detection of processed pseudogenes}

To determine if a pseudogene could arose from a retrotransposition event, we systematically checked for classical retro-transposition hallmarks: lack of intron with respect to their paralog CDS, retrotransposon-related sequences within the first flanking annotated elements of the pseudogene (one upstream and one downstream), and polyA-tail at the 3 '-end of the pseudogene. For this last check, we analyzed the A content of the 3 '-end flanking region (500 nucleotides) of each pseudogene. We considered as a potential poly(A)-tail, a window of 50 nucleotides containing at least 35 adenines, with at least one stretch of 5 adenines, and less than 10 thymines. To avoid fortuitous signals due to AT-rich sequences, the same procedure was performed on 1000 random sequences (with identical nucleotide composition). Only sequences for which no signal was detected in the random sequences were considered as potential poly(A)-tail.

\section{Statistical analysis}

Correlation tests were done with the method of Spearman implemented in $\mathrm{R}$ [36]. We accepted a correlation between data sets when the p-value was lower than 0.05 .

\section{Results}

\section{Strategy}

We systematically searched for all potential pseudogenes of ancient protein-coding genes within 8 completely sequenced and annotated genomes of Hemiascomycetes: Saccharomyces cerevisiae and Candida glabrata (two Saccharomycetaceae that underwent ancient polyploidization), Zygosaccharomyces rouxii, Kluyveromyces lactis, Kluyveromyces thermotolerans, Saccharomyces kluyveri (four protoploid Saccharomycetaceae), and Debaryomyces hansenii and Yarrowia lipolytica, members of the «CTG» group and of the Dipodascaceae, respectively (http://www.genolevures.org). To do this, we compared each genome sequence to the set of 44,174 protein-coding sequences (CDS) annotated in these genomes (see Methods). We considered here as pseudogene any sequence that simultaneously -i- does not overlap an already annotated genetic element, -ii- shares sequence similarity with an annotated CDS (either in the same genome or in one or several other yeast genomes considered) and -iii- shows disabling mutations in the reading frame: in-frame stop codon, frameshift mutation or truncation of more than $30 \%$ relatively to the CDS. We chose this limit of $30 \%$ because, among the functional members of a given protein coding gene family, the length variation does not exceed $30 \%$ in the majority of cases (data not shown). Inactive pseudogenes resulting solely from mutations in promoters, as well as pseudogenes without detectable similarity among the eight studied genomes are, therefore, excluded from our analysis. Simi- 
larly, we do not question the genome annotations: an annotated gene that appears truncated compared to other homologous sequences will not be considered as a pseudogene because truncation does not necessarily imply inactivation (experimental work is needed to precise such a point).

\section{Complete set of detected pseudogenes and sequence degradation patterns}

We found a total of 871 pseudogenes among the 8 yeast genomes (see Additional file 1, Table S1 for complete list and Methods for nomenclature). They correspond to 418 distinct protein families or singletons. The total number of pseudogenes varies significantly between species: from 38 in C. glabrata to 230 in Y. lipolytica, corresponding to only $0.7 \%$ and $3.6 \%$ of the annotated CDS, respectively (Table 1).

The pseudogenes identified exhibit a large panel of sequence degradation, ranging from only a few mutational disablements to extensive truncations. We distinguished here the «full-size» pseudogenes, i.e. those extending over more than $70 \%$ of their closest functional homolog, from the «truncated» pseudogenes (Table 1).

In $S$. cerevisiae, S. kluyveri, $K$. thermotolerans and $K$. lactis, the number of «full-size» pseudogenes exceeds truncated ones. The opposite is true for C. glabrata, $Z$. rouxii, $D$. hansenii and $Y$. lipolytica. There are as many pseudogenes truncated from their 3 '-end, as those truncated from their 5 '-end (Table 1), consistent with the idea that truncation results from deletion at the DNA level rather than incomplete cDNA formation, characteristic of retro-processed pseudogenes. «Full-size» pseudogenes contain more disabling mutations (in-frame stop codons and frameshifts) than truncated pseudogenes (except in
D. hansenii and Y. lipolytica) (Additional file 1, Table S1). We found four pseudogenes (DEHA2Bp3, DEHA2Cp3, $D E H A 2 D p 5, D E H A 2 E p 5)$ inactivated by insertion of a DNA fragment of mitochondrial origin (NUMTs), as previously described [37].

\section{Sequence divergence between pseudogenes and their best functional homologs}

In the absence of the actual ancestral coding sequences of the pseudogenes, we estimated the distances between pseudogenes and their closest functional homologs (bestmatches) to measure their degree of mutational decay (Additional file 1, Table S1; see Methods for details). Distributions of the p-distances were calculated for each yeast species (figure 1). They range from 0 to 0.8 globally. The distributions are different if one considers pseudogenes whose bestmatch is not in the same genome and pseudogenes whose bestmatch is a paralog in the same genome. In the first case, distances are high, consistent with the generally long evolutionary distances between studied species. In the second case, distances are much lower than that observed between functional paralogs in the same genome, suggesting that many pseudogenes arose after recent gene duplication events. The genomes of S. kluyveri and K. thermotolerans contain the highest proportions of highly diverged pseudogenes (half of them differ by more than $60 \%$ from their bestmatches), while the genomes of S. cerevisiae and Y. lipolytica contain the highest proportions of less diverged pseudogenes (half of them differ by less than $28 \%$ from their bestmatches). Note that functional paralogs (right panel) in all these genomes are issued from both ancient (prior to the speciation events) and recent (species-specific) duplications [25].

Table 1: Number and types of pseudogenes identified in the eight yeast genomes studied.

\begin{tabular}{|c|c|c|c|c|c|c|}
\hline Species & Genome size ${ }^{a}$ & Total CDS & Pseudogenes $^{b}$ & " Full-size ${ }^{c}(\geq 70 \%)$ & $\begin{array}{c}\text { 3'-Truncationd } \\
(<70 \%)\end{array}$ & $\begin{array}{c}\text { 5'-Truncatione } \\
(<70 \%)\end{array}$ \\
\hline S. cerevisiae & 12.1 & 5769 & $77(1.3)$ & 0.57 & 0.21 & 0.22 \\
\hline C. glabrata & 12.3 & 5204 & $38(0.7)$ & 0.47 & 0.32 & 0.21 \\
\hline Z. rouxii & 9.8 & 4998 & $105(2.1)$ & 0.42 & 0.28 & 0.30 \\
\hline K. thermotolerans & 10.4 & 5104 & $68(1.2)$ & 0.54 & 0.16 & 0.29 \\
\hline S. kluyveri & 11.3 & 5308 & $117(1.3)$ & 0.63 & 0.19 & 0.18 \\
\hline K. lactis & 10.7 & 5084 & $61(2.2)$ & 0.66 & 0.18 & 0.16 \\
\hline D. hansenii & 12.2 & 6273 & $175(2.8)$ & 0.37 & 0.28 & 0.35 \\
\hline Y. lipolytica & 20.5 & 6434 & $230(3.6)$ & 0.36 & 0.3 & 0.35 \\
\hline
\end{tabular}

a. in Megabases, except rDNA.

b. Pseudogenes of protein coding sequences only. The percentage of pseudogenes relative to CDS is indicated in parenthesis.

c. Proportion of « full-size » pseudogenes, i.e. extending over more than $70 \%$ of their bestmatch length.

d. Proportion of pseudogenes extensively truncated at their 3'-end.

e. Proportion of pseudogenes extensively truncated at their 5'-end. 




Pseudogenes across species, conservation or independent formation

A pseudogene should be free from any functional pressure and, therefore, should not be conserved over long evolutionary periods, unless it acquires a functional role. We, therefore, searched for pseudogenes conserved in several yeast species as a possible functional signature. A total of 274 pseudogenes are shared between at least two species but 263 of them are located outside of any conserved synteny block and probably correspond to independent pseudogenization events of homologous genes in different lineages. The remaining 9 pseudogenes are found among five conserved synteny blocks, defining five groups of orthologous pseudogenes (Figure 2). Note that 


S.cerevisiae
$\begin{aligned} & \text { C. glabrata } \\ & \text { Z. rouxii }\end{aligned}$

we did not consider the syntenic orthologs of YILO09C-A (EST3), YPL052W (OAZ1) and YOR239W (ABP140) as pseudogenes, because they all bear the programmed translational frameshift present in the functional genes of S. cerevisiae [38]. According to the species phylogeny and following parsimony criterion, three of these sets of orthologous pseudogenes also correspond to independent pseudogenization events of the same gene in distinct lineages (Set 3, 4 and 5 on Figure 2).

Pseudogenes SAKLOGP7 and KLTHOGp3, corresponding to YDR285W (ZIP1) of S. cerevisiae are conserved between S. kluyveri and K. thermotolerans. ZIP1 is a transverse filament protein of the synaptonemal complex that is required for normal levels of meiotic recombination and pairing between homologous chromosomes during meiosis. The 2 orthologous pseudogenes are «fullsize» and share a single common frameshift mutation (data not shown). The most parsimonious hypothesis is that the frameshift mutation appeared in the common ancestor of these 2 species.

REC107 is a meiotic gene in S. cerevisiae, which has been lost several times in hemiascomycetes [39]. This gene is also pseudogenized in three of the newly sequenced genomes studied. SAKLOGP13 and KLTHOBp 3 are conserved between $S$. kluyveri and $K$. thermotolerans, suggesting a pseudogenization event in their common ancestor. The orthologous gene is also pseudogenized in Z. rouxii (ZYROOGp9), and probably corresponds to another independent event. Unlike in the previous case, the pseudogenes of this block of conserved synteny are truncated and each copy accumulated several species-specific disabling mutations.

\section{Conservation of pseudogenes across S. cerevisiae strains}

The genome sequences for the S. cerevisiae non-reference strains are draft assemblies, not suitable for the pseudogene detection procedure developed in this work. How- ever, to get a first estimation of pseudogene conservation among strains, we examined the conservation of the pseudogenes identified in the $S$. cerevisiae reference strain S288C (Additional file 1, Table S1) among 7 other sequenced strains (see Methods). We found that 62 of the pseudogenes identified in S288C are also pseudogenized in all other strains, suggesting that the pseudogenization event occurred in their common ancestor. The 14 other pseudogenes of S288C sometimes correspond to intact coding sequences in one or a few other strains, indicating more recent pseudogenization events (Table 2). 11 of these 14 pseudogenes contain at least two degrading mutations (in-frame stop codon or frameshift mutation) and 8 of them are truncated at their $3^{\prime}$ or 5 ' end. The only pseudogene specific to S288C (SACEOIp4) contains only one internal stop codon.

\section{Distribution of the pseudogenes among protein families and corresponding functions}

Based on sequence similarity, pseudogenes can be attributed to the functional gene families classified according to their predicted translational products [27]. Such families contain groups of orthologs between species, as well as groups of paralogs resulting from gene duplications and losses (see [40] for a review). In each yeast species, the majority of pseudogenes (from 51 to $88 \%$ ) belong to gene families with a functionally characterized S. cerevisiae member, which can be used to infer the probable function of the gene that was pseudogenized. Interestingly, we observed a bias towards transporters, proteins acting at the periphery of the cell, and enzymes (Table 3). When normalized against the ratios of these functional categories among active genes, the bias is conserved for transporters and proteins acting at the periphery of the cell, but not for enzymes. Among pseudogenes with no ascribed function, there is no over-representation of domains related to these three categories. Although the 
Table 2: Pseudogenes in S. cerevisiae S288C with non-degraded homologs in other S. cerevisiae strains.

\begin{tabular}{|c|c|c|c|c|c|c|c|}
\hline S288C & YJM789 & RM11_1A & YPS163 & M22 & EC1118 & JAY291 & AWRI1631 \\
\hline SACEOIp6 & $P$ & $P$ & $\mathrm{p}$ & $P$ & $p$ & intact & Intact \\
\hline SACEOJp5 & $\mathrm{P}$ & $P$ & $p$ & $\mathrm{P}$ & $p$ & intact & Intact \\
\hline SACEOAp2 & $P$ & $P$ & $\mathrm{p}$ & $P$ & $\mathrm{p}$ & $P$ & Intact \\
\hline SACEODp6 & $\mathrm{P}$ & $\mathrm{P}$ & intact & intact & $p$ & intact & Intact \\
\hline SACEOIp2 & $P$ & $P$ & $\mathrm{p}$ & $P$ & intact & intact & Intact \\
\hline SACEOIp4 & Intact & intact & intact & intact & intact & intact & Intact \\
\hline SACEOJp1 & $P$ & $P$ & $\mathrm{p}$ & $P$ & intact & intact & Intact \\
\hline SACEOOp3 & $\mathrm{P}$ & $\mathrm{P}$ & intact & $\mathrm{P}$ & intact & intact & Intact \\
\hline SACEOOp2 & $P$ & $P$ & $p$ & $P$ & intact & intact & $P$ \\
\hline SACEOAp 9 & Intact & $P$ & $\mathrm{p}$ & $P$ & intact & $P$ & $P$ \\
\hline SACEOOp6 & $P$ & $P$ & $\mathrm{p}$ & $P$ & intact & $P$ & $P$ \\
\hline SACEOFp3 & $\mathrm{P}$ & $\mathrm{P}$ & $\mathrm{p}$ & $P$ & $\mathrm{p}$ & intact & $P$ \\
\hline SACEOAp 11 & $\mathrm{P}$ & $P$ & $\mathrm{p}$ & intact & $\mathrm{p}$ & $\mathrm{P}$ & $P$ \\
\hline SACEOCp2 & $P$ & $P$ & $\mathrm{p}$ & intact & $\mathrm{p}$ & $\mathrm{P}$ & $P$ \\
\hline SACEOGp 1 & $\mathrm{P}$ & $P$ & $p$ & intact & $p$ & $P$ & $\mathrm{P}$ \\
\hline
\end{tabular}

p: homologous pseudogene, intact: homolog with non-degraded coding sequence.

number of pseudogenes is not directly correlated to the number of its active paralogs in a given family, we observed frequent formation of pseudogenes in these functional categories. For example, there are11 pseudogenes in $D$. hansenii for the sugar transporter family, 12 pseudogenes in C. glabrata for the lectin-like protein family, and 25 pseudogenes in Z. rouxii for the DUP240 gene family coding for membrane proteins (Additional file 1, Table S2).

A total of 139 pseudogenes are similar to singleton genes, and 288 (one third of total) belong to gene families specific to a given species for which, unless in S. cerevisiae, there is usually no functional indication (Additional file 1, Table S2).

\section{Clustering of the pseudogenes in subtelomeres}

Gene densities are nearly constant along yeast chromosomes, except for subtelomeres where the number of active genes is reduced [41], and where genes can be transcriptionally silenced [42]. We, therefore, examined the distribution of the pseudogenes along the chromosomes, separating the subtelomeres (30 kilobases apart from the telomeres or from sequenced chromosome ends) from

Table 3: Repartition of the pseudogenes according to the presence/absence of an S. cerevisiae homolog, and their functional classification



S.c. is for S. cerevisiae.

aNumber of pseudogenes with a homolog in S. cerevisiae coding for proteins involved in transport and/or acting at the periphery of the cell.

bNumber of pseudogenes without homolog in S. cerevisiae coding for enzymes. 
the central regions (Table 4). In all yeast species, pseudogenes exceed active genes in number ( 3 to 16 times more) in the subtelomeres. In S. cerevisiae, C. glabrata and Z. rouxii, more than half of the pseudogenes are found in subtelomeres (70, 60 and $54 \%$, respectively). This proportion is lower in $K$. thermotolerans, D. hansenii, S. kluyveri and $K$. lactis (47, 40, 36 and 30\% respectively), and drops to only 5\% in Y. lipolytica. The presence of rDNA loci in the subtelomeres of $Y$. lipolytica (6 loci) might prevent some pseudogenes accumulating in these regions, as well as in D. hansenii (3 loci) and in C. glabrata (2 loci) [25].

\section{Possible origin of the pseudogenes}

We tried to define the origin of yeast pseudogenes based on the presence or absence of a paralog in the same genome and on the conservation of synteny between species (figure 3). The presence of an active paralog in the genome reveals the occurrence of a previous duplication event, followed by mutational inactivation of one of the 2 copies. The absence of any paralogs reveals the degradation of a single copy gene, hence the loss of the corresponding function in the species. The conservation of synteny is a signature for mutational sequence degradation at the origin of the pseudogene. The non-conservation of synteny is compatible with a species-specific duplication, either a retro-transposition event or a segmental duplication.

\section{Pseudogenes originating after species-specific gene duplications}

More than half of the pseudogenes arose after a gene duplication event specific to their own genome (Table 5). This corresponds to the vast majority of pseudogenes in S. cerevisiae, D. hansenii and Y. lipolytica (96, 87 and 93\%, respectively), ca. $70 \%$ of the pseudogenes in C. glabrata and $Z$. rouxii, and less than $60 \%$ in $K$. lactis, $K$. thermotolerans and S. kluyveri. The majority of these pseudogenes probably arose after segmental duplication, but some may be processed pseudogenes, as suggested by a poly(A) tract at their 3'-end or their location next to a retrotransposon-related sequence [43]. This would correspond to only $3.5 \%$ of the pseudogenes in Y. lipolytica, and $20 \%$ of the pseudogenes in K. thermotolerans. Only one pseudogene (DEHA2Ep25) was identified by the lack of intron compared to its closest functional homolog.

Interestingly, we found duplications of pseudogenes, as evidenced by their common pattern of degrading mutations. For example, in Y. lipolytica, we found five pseudogenes similar to YALIOA14927g (Figure 4), which, based on phylogenetic analysis, suggest two original pseudogenization events in the history of this family. Note that these pseudogenes are not located in subtelomeric regions and are not part of larger duplicated regions including other genes. These pseudogenes have thus not been maintained in the genome by some selective pressure on the duplication of adjacent genes. Similarly, two such cases of pseudogene multiplication were encountered in K. lactis, as well as 12 cases of pseudogene duplication (Additional file 1, Table S1).

Most pseudogenes located at the chromosome ends arose after a species-specific gene duplication (Table 6): from $60 \%$ in $K$. thermotolerans to $100 \%$ in Y. lipolytica.

\section{Pseudogenes originating from ancestral gene duplications}

Pseudogenes formed after ancestral gene duplications are found in all yeast genomes (Table 5). Four pseudogenes, SACEOBp1, CAGLODp2, CAGLOHp3 and CAGLOMp5 were formed after the whole-genome duplication event that occurred in the common ancestor of C. glabrata and S. cerevisiae [44] (Figure 5). This is a very small number

Table 4: Subtelomeric localization of pseudogenes and presence/absence of annotated paralogs

\begin{tabular}{|c|c|c|c|c|c|}
\hline \multirow[t]{2}{*}{ Species } & \multirow[t]{2}{*}{ P. endsa } & \multirow[t]{2}{*}{ G. ends $s^{b}(\%)$} & \multirow[t]{2}{*}{ No paralogc } & \multicolumn{2}{|l|}{ Paralogd } \\
\hline & & & & In & Out \\
\hline S. cerevisiae* & 71.4 & 5.4 & 3 & 67 & 7 \\
\hline C. glabrata & 60.5 & 3.2 & 8 & 27 & 3 \\
\hline Z. rouxii & 54.3 & 3.2 & 14 & 70 & 21 \\
\hline K. thermotolerans & 48.5 & 2 & 18 & 39 & 11 \\
\hline S. kluyveri & 37.6 & 2.6 & 29 & 57 & 31 \\
\hline K. lactis* & 31.1 & 2.9 & 14 & 33 & 14 \\
\hline D. hansenii & 40.0 & 2.7 & 8 & 151 & 16 \\
\hline Y. lipolytica & 5.2 & 1.6 & 6 & 221 & 3 \\
\hline
\end{tabular}

a. Percentage of pseudogenes in subtelomeric regions (less than $30 \mathrm{~kb}$ from a chromosome end).

b. Percentage of active genes in subtelomeric regions.

c. Number of pseudogenes without annotated functional paralog in the genome.

d. Number of pseudogenes with annotated functional paralog whose closest homolog is in the same genome (in) or in another genome (out).

*. Species for which all chromosomes are fully sequenced, including their telomeric repeats. 




Figure 3 Possible origin of pseudogenes. See text for explanations. The diamonds correspond to distinctive criteria and rectangles to deduced origin.

compared to the extensive loss of duplicated genes that occurred by deletion [45]. Strikingly, there are more pseudogenes originated from other ancestral duplications in protoploid Saccharomycetaceae and in D. hansenii and $Y$. lipolytica, suggesting that selective pressure on duplicated genes are different after whole-genome duplication and other duplication events, such as segmental duplications.

Pseudogenes originating without previous gene duplication and putative loss of function

Pseudogenes with no functional paralog (Additional file 2, Table S3) are found in all the studied genomes and could, therefore, correspond to a functional loss. The genomes of $K$. lactis, $K$. thermotolerans, S. kluyveri and $C$. glabrata contain the highest proportions of such pseudogenes (23\%, 26\%, 25\% and 20\%, respectively). The lowest proportions are found in D. hansenii and in Y. lipolytica (5\% and 3\%, respectively), consistent with the higher gene redundancy in these genomes [26]. There is no bias towards any functional category (Additional file 2, Table S3). Pseudogenes homologous to essential genes in S. cer- evisiae are found in all species, except in $K$. lactis. Repeated pseudogenization of the same gene is also encountered in this category. For example, pseudogenes CAGLOMp1 and ZYROOGp11, similar to the essential chromosomal passenger gene YJR089W (BIR1), have no homolog in their respective genome.

Finally, we noticed three interesting pseudogenes in $S$. cerevisiae that correspond to horizontally acquired genes. SACEOFp3 and SACEOFp2 have no homolog in S. cerevisiae but were identified by similarity to DEHA2D01122g (similar to a bacterial tryptophan synthase) and DEHA2E07282g (similar to a bacterial glyoxalase), respectively. Absence of these genes among hemiascomycetes suggests that four independent horizontal gene transfer events have occurred, the two genes in S. cerevisiae being secondarily pseudogenized. The third one, $S A C E O N p 2$ is a duplicated and identical copy of $S A C E O F p 2$, revealing the expansion of horizontally transferred inactivated genes. The transfers must have occurred in an ancestor of all the studied strains of $S$. cerevisiae because the homologs of SACEOFp3, SACEOFp2 
Table 5: Classification of pseudogenes according to their possible origin.

\begin{tabular}{|c|c|c|c|c|c|c|}
\hline Species & $\begin{array}{l}\text { Species-specific } \\
\text { duplication }^{\text {a }}\end{array}$ & Ancestral duplicationb & Function lossc & $\begin{array}{l}\text { duplicated } \\
\text { pseudos }^{\text {d }}\end{array}$ & $\begin{array}{l}\text { duplicated } \\
\text { segmente }\end{array}$ & retros $f$ \\
\hline S. cerevisiae & 73 & 1 & 3 & 1 & 0 & 1,6 \\
\hline C. glabrata & 27 & 3 & 8 & 1 & 1 & 3,0 \\
\hline Z. rouxii & 71 & 20 & 14 & 6 & 41 & 1,3 \\
\hline K. thermotolerans & 39 & 11 & 18 & 0 & 17 & 0,16 \\
\hline S. kluyveri & 65 & 23 & 29 & 4 & 13 & 1,12 \\
\hline K. lactis & 35 & 12 & 14 & 5 & 7 & 0,4 \\
\hline D. hansenii & 153 & 14 & 8 & 2 & 62 & 1,3 \\
\hline Y. lipolytica & 213 & 11 & 6 & 6 & 19 & 2,8 \\
\hline \multicolumn{7}{|c|}{$\begin{array}{l}\text {. number of pseudogenes originating from mutational inactivation of a duplicated gene copy formed after speciation. } \\
\text { b. number of pseudogenes originating from mutational inactivation of a duplicated gene copy formed before speciation. } \\
\text {. number of pseudogenes originating from mutational inactivation of a single copy gene. } \\
\text { d. number of duplicated pseudogenes among the first category (a). } \\
\text { e. number of pseudogenes being part of a duplicated segment involving other adjacent genes among the first category (a). } \\
\text { f. number of retro-processed pseudogenes, among the first category (a), identified by: either the presence of a 3' poly(A)-tail (first number) or } \\
\text { the proximity of retrotransposon-related sequence (second number). In each species, the candidates identified by these } 2 \text { criteria are } \\
\text { different. }\end{array}$} \\
\hline
\end{tabular}

and $S A C E O N p 2$ are pseudogenized in the 7 other strains examined.

\section{Discussion}

We present here the first systematic search for pseudogenes in the genomes of eight distinct yeast species spanning the whole evolutionary spectrum of hemiascomycetes. Because our method of detection relies on sequence similarity with annotated protein coding genes, the total number of pseudogenes identified (871) represents a minimal estimate (pseudogenes without any functional homolog or with alteration of their promoter sequences were not examined). Among the genomes analyzed here, only $S$. cerevisiae has been the subject of previous systematic analysis of the pseudogene content $[6,7]$. Our analysis revealed a smaller number of pseudogenes in this species (77) because we deliberately ignored partial overlaps with annotated genetic elements. On the contrary, additional pseudogenes were identified based on our multi-species sequence comparisons. These differences illustrate the difficulties in listing all pseudogenes in a given genome. Despite this fact and numerical variations between yeast species, our results demonstrate that the proportion of pseudogenes compared to active genes remains low in all hemiascomycetous genomes studied (comparable to the proportion of pseudogenes in D. melanogaster and much smaller than the proportions in mammalian genomes, see http://pseudogenes.org).

Our comparative analysis of pseudogenes across an entire yeast phylum provides a unique data set to examine their origin and evolutionary conservation. We found that pseudogenes in yeasts are formed either by disabling mutations (in-frame stop codons and frameshift mutations) or by extensive truncations. The general absence of conservation of pseudogenes between yeast species is consistent with their large evolutionary distances $[25,26]$ and indicates that new pseudogenes were formed within each lineage. However, intra-species conservation of pseudogenes is high: about $80 \%$ of the pseudogenes in the $S$. cerevisiae reference strain are old enough to be conserved among 7 other strains of this species. Poor conservation of pseudogenes is also observed between mammalian species [46,47]. Most of the pseudogenes correspond to duplicated gene copies, illustrating the extensive dynamics of gene duplications in the yeast genomes, most probably segmental duplications, as observed in S. cerevisiae [48]. Only few pseudogenes correspond to duplicated gene copies formed by the ancestral whole-genome duplication common to C. glabrata and $S$. cerevisiae [44]. This is consistent with the idea that most duplicated copies were lost by complete deletion, as previously proposed $[45,49]$, or with the possibility that pseudogenes have been degraded beyond recognition, given the time elapsed since the whole-genome duplication event. Among the protoploid Saccharomycetaceae, the presence of species-specific pseudogenes within conserved synteny blocks indicates their relatively recent formation, to the notable exception of two ancestral pseudogenes conserved in S. kluyveri and K. thermotolerans, the more closely related pairs of species studied. The general absence of conserved pseudogenes confirms that 
a)

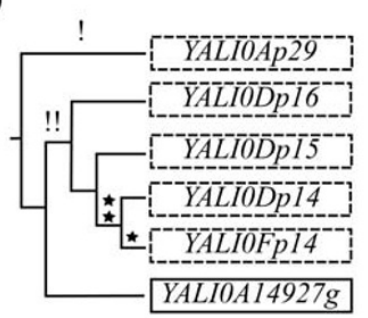

b)

YALIOAp29
YALIODP16
YALIODP15
YALIODP14
YALIOFP14
YALIOA14927g

YALIOAP29

YALIOA14927g

YALIOAp29

YALIODP16

YALIODP15

YALIODP14

YALIOFP1 4

YALIOA $14927 \mathrm{~g}$

101

YALIOAp29

YALIODP16

YALIODP15

YALIODP1 4

YALIOFP1 4

YALIOA $14927 \mathrm{~g}$

YALIOAP29

YALIODP16

YALIODP 15

YALIODP 14

YALIOFP14

YALIOA14927g

51

151
1

FHLRAVVIVSQCHYCA-GI]!LNVSQLLQRVLLWWLVEFHHRAAPGGFSAL FTESSAGITCRFTVSLAGI]!-NVFQSLQRLLLWWLVEFHHRAAPGGFSAL FHLRAVVIVSQCHSCA-GI|!NVSQSLQRLLLWWLVEFHHRAAPGGFSAL FHLRAVVIVSQCHSCA-GI)!LNVSQSLQRLLLWGLVEFHHRAAPGGFSAL MVLKFCSCSSQFLLCAVVIIDVFQA-LQHLLLWWLVEFHLRAAPGGFSAL

S! FCCWHLSVGGSSSGSLRLGLALCSGGLAGWAQTLCSVAVFADAYAQÄ S! FCCRHLSVGGSSSGSLRPGLALCSGGLAGWAQTLCSVAVFADAYAQAE S! FCCRHLSVGGSSSGSLRPGLALCSGGLAGWAQTLCSVAVFADAYAQAE S! FCCRHLSVGGSSSGSLRPGLALCSGGLAGWAQTLCSVAVFADAYAQAE Fo-CC---VVGRWLSGSLRLGLVLCSGGLAGWAQTLCSMAVFVDS--NAK

KAGSGSDRVSAVLLTVIPGVLIPSQQQMGETYDCSNDSPSPQSIQELLSM QAGSGSDRGSAVLLTVIPGVLIPSQQOMEKTYDCSNDIPSLOSIQELLFM QAGSGSDRGSAVLLTVIPGVMIPSQQQMEKTYDCSNDSPSLQSIQELLSM QAGSGSDRGSAVLLTVIPGVLIPSQQQMEKTYDC *NDSPSLQSIQELLSM QAGSGLVRVPAVLLTVIAGVLILSQQQMEGTYDCSNDSPSLQSIQELLSM

QGVAPQGATHTFG!LMDNVSCSSQLHIHGDRILEGLVNPPTRHHPAFS QVVAPKGATHTVGELMDNVPCPSLLHIHGDRIHEGLAKSPTRHHPASS QGIAPKGATHTFGELMDNVSCPSQLHIHGDRIHERLAKPPTRHHPASS QGIAP * GATHTLGELMDNVSCPSQLQIHGDRIHEWLAKPPTRHHPASS QGIAP *GATHTFGELMDNVSCPSQLHIHGDRIHERLAKPPTRHHPASS QGVAP KATHTVGELLDNVSCPSLLHIHGDRIHEGLAKPPTRHHPASS

Figure 4 Scenario for the multiplication of pseudogenes in $Y$. lipolytica. a) Rectangle represents the functional gene, dashed rectangles represent its corresponding pseudogenes. The tree topology is obtained by maximum likelihood reconstruction [69] based on the aligned nucleic acid sequences (branch lengths ignored). The emergence of frameshift mutations (!) and in-frame stop-codons (*) are indicated above corresponding branches. b) Alignment of the translation products of YALIOA14927g and its pseudogenes obtained by MUSCLE. frameshift mutations (!) and in-frame stop-Codons $(*)$ are boxed.

most of the observed pseudogenes are on their way to complete degradation.

According to Doniger et al., [32] 49 pseudogenes containing an internal in-frame stop codon (based on our own criteria) and 5 pseudogenes containing a frameshift mutation are present in either M22 or YPS163, while they correspond to intact coding sequences in S288C. From the alignment of the three genomes within coding regions, it appears that the loss of a gene by deletion is much more frequent (3 deleted genes for 19 large indels ( $>100 \mathrm{bp})$ ) than the creation of a pseudogene by a substitution event (49 pseudogenes for 46807 SNP) or by an indel event (5 pseudogenes for 960 small indels $(<100$ $\mathrm{bp})$ ). According to Lynch et al. [50], the rate of gene loss, $2.1 \times 10^{-6}$ per gene per cell division, is much higher than the base-substitutional rate, $0.33 \times 10^{-9}$ per site per cell division. It should then be much more frequent to lose a gene by deletion than to create a pseudogene.

Table 6: Number subtelomeric pseudogenes according to their possible origin.

\begin{tabular}{|c|c|c|c|}
\hline Species & Species-specifica & Ancestral & Function loss \\
\hline S. cerevisiae & 49 & 1 & 3 \\
\hline C. glabrata & 18 & 0 & 5 \\
\hline Z. rouxii & 45 & 7 & 5 \\
\hline K. thermotolerans & 32 & 8 & 6 \\
\hline S. kluyveri & 20 & 7 & 4 \\
\hline K. lactis & 16 & 2 & 1 \\
\hline D. hansenii & 67 & 4 & 0 \\
\hline Y. lipolytica & 11 & 0 & 0 \\
\hline
\end{tabular}

See Table 5 for legend. 


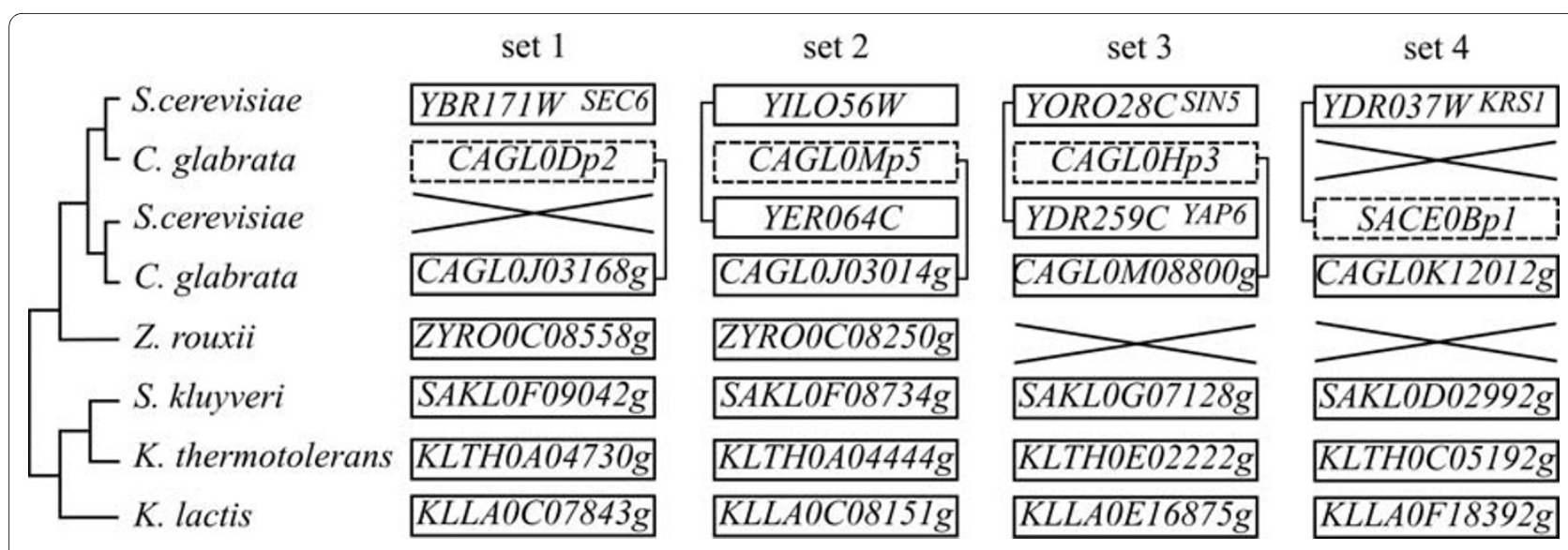

Figure 5 Pseudogenes in pairs of ohnologs in Saccharomycetaceae. Same legend as Figure 2. Pairs of ohnologs, i.e. paralogs originating from the whole-genome duplication [70], are linked by brackets.

Unlike their abundance in mammalian genomes $[15,51]$, only few pseudogenes may have originated from retro-transposition events in yeasts. But their presence suggests that, as experimentally demonstrated in S. cerevisiae $[43,52,53]$, retro-transposition occurred in these genomes, although a very small number of active retrotransposons are usually present $[25,54]$. The number of retro-processed pseudogenes in yeasts is, however, probably underestimated because their identification is difficult given the small number of intron-containing genes. The few detected pseudogenes homologous to introncontaining genes are all truncated and do not span the intron insertion-site, except in one case (DEHA2Ep25).

Pseudogenes without functional paralog suggest functional loss in the corresponding species, unless a nonhomologous gene encodes a similar function [55]. In $S$. cerevisiae, the fact that about half of the pseudogenes correspond to unknown functions is striking given that there remain only $17 \%$ of the genes not yet functionally characterized in this species [56]. This suggests that the divergence of functional repertoire between yeast species primarily concerns functions not yet identified. The frequent occurrence of pseudogenes corresponding to transporters or protein acting at the periphery of the cell may be correlated with the tendency of such genes to cluster in the subtelomeric regions, which are highly dynamic in terms of gene duplications and losses $[38,41,57]$ and often concern functions involved in the adaptation of the species to its environment $[22,58]$. The highly dynamical behavior of such families is supported by the fact that 3 to 16 times more pseudogenes than genes are found in the subtelomeric regions. The vast majority of pseudogenes in the subtelomeres originated from a species-specific duplication. This bias could be correlated to the accelerated base-pair substitution observed in the subtelomeres, which probably also contributes to adaptive evolution [59]. It also suggests that pseudogenes issued from ancestral duplication and function loss are not preferentially maintained in subtelomeres with respect to central regions of chromosomes.

In $S$. cerevisiae, pseudogenes of highly connected genes are significantly under-represented, possibly reflecting the lower propensity of gene loss among these genes: $6 \%$ of the pseudogenes of genes coding for protein in complexes against $28 \%$ of the active genes [60] and $59 \%$ of pseudogenes of genes with a genetic interaction profile against $75 \%$ of the active genes [61].

Whether pseudogenes correspond to intermediate gene states before complete erasure or steady states conferring selective advantages remains an open question and may be case-specific. Our data provide no indication as to whether pseudogenes may be transcribed or not. However, according to available experimental data in S. cerevisiae (oligonucleotide tiling array experiments [62], 3'-long SAGE approach [63], and direct RNA sequencing [64]), 12 of the 77 detected pseudogenes appear to be transcribed (Table 7). Evidence of transcription also exists in C. glabrata and Y. lipolytica (H. Müller, personal communication; C. Neuvéglise, personal communication). The transcription products of pseudogenes may be directly targeted to the degradation machinery such as NMD [65], but they could also play some role in the cell by interfering with the expression of functional genes. Moreover, as previously suggested [6], pseudogenes with few disabling mutations could constitute a reservoir of functional protein products if recoding events occur, such as programmed frameshift (See [38] for a comparative analysis of the programmed frameshifting in Saccharomycetales), or bypass of the stop-codon [66].

\section{Conclusions}

Pseudogenes are found in all yeast genomes, albeit in limited number compared to genomes of multi-cellular eukaryotes. They mostly result from lineage-specific 
Table 7: Pseudogenes with evidence of transcriptionin S. cerevisiae

\begin{tabular}{|c|c|c|c|c|}
\hline Name & stops & frameshifts & R.L & Reference \\
\hline SACE0Ap 1 & 0 & 1 & 1 & [63] \\
\hline SACE0Ap2 & 1 & 0 & 0.9 & [63] \\
\hline SACE0Ap7 & 1 & 4 & 0.11 & [63] \\
\hline SACE0Ap 13 & 0 & 1 & 1 & [63] \\
\hline SACE0Bp1 & 5 & 15 & 1 & [62-64] \\
\hline SACE0Cp2 & 2 & 0 & 0.06 & [63] \\
\hline SACEOCp3 & 10 & 7 & 1 & [63] \\
\hline SACE0Dp6 & 1 & 0 & 0.82 & {$[62,63]$} \\
\hline SACEODp7 & 5 & 11 & 0.57 & [63] \\
\hline SACEOHp1 & 1 & 8 & 0.86 & {$[62]$} \\
\hline SACE0Lp4 & 0 & 0 & 0.55 & [64] \\
\hline SACEOPp4 & 1 & 2 & 0.77 & [62] \\
\hline
\end{tabular}

The number of disabling mutations within each pseudogene is given in columns 2 and 3. The relative length (R.L) of the pseudogene with respect to its closest functional homolog is indicated in column 4. Last column indicates the reference of the data set where evidence of transcription is found (see text for details). For a given chromosome, all identified pseudogenes are separated by several genes. There is no bias of any kind among these pseudogenes.

mutational degradations that may correlate with species adaptation to their environment. Yeast pseudogenes show a wide range of mutational alterations, consistent with their rapid evolution, hence their absence of conservation between species. Along with complete gene deletion, pseudogene formation contributes to the rapid genome evolution by gene duplication and loss in yeasts. The paucity of observed pseudogenes across the entire phylum of Hemiascomycetes suggests that pseudogene formation is not the main mechanism of gene loss within these genomes. This could be explained by the low estimated rate for pseudogene formation across $S$. cerevisiae strains, compared to the estimated rate of gene deletion. However, despite their unlikely occurrence, pseudogenes do exist in the yeast genomes. They appear mainly by species-specific duplications and testify for the adaptation of the cell to its environment. Their poor conservation across species suggests that most of them are likely to disappear.

\section{Additional material}

Additional file 1 Table S1 and Table S2. - Table S1. List and characteristics of the detected pseudogenes in the 8 studied yeast genomes. - Table S2. List of gene families (or singletons) with detected pseudogenes.

Additional file $\mathbf{2}$ Table S3 and descriptions of Tables S1 and S2.

Descriptions of Tables S1 and S2. - Table S3. Lost functions among the eight studied species. A list of the pseudogenes with no paralog in the different genomes

\section{Authors' contributions}

IL designed and conducted the analysis, implemented the algorithms and wrote the manuscript. BD contributed to the interpretation of the results and to the writing of the manuscript. Both authors read and approved the final manuscript.

\section{Acknowledgements}

We thank Gilles Fischer, Bertrand Llorente and Eduardo Rocha for helpful comments on this work and our colleagues from the "Unité de Génétique Moléculaire des Levures" and from the Génolevures consortium for fruitful discussions. This work was supported by grant ANR-05-BLAN-0331 from the Agence Nationale de la Recherche (ANR). BD is a member of the Institut Universitaire de France.

\section{Author Details}

1 Unité de Génétique Moléculaire des Levures, Institut Pasteur, Paris, France, 2Unité de Formation et de Recherche 927, Université Pierre et Marie Curie, Paris, France and 3 Unité de Recherche Associée 2171, Centre National de la Recherche Scientifique, France

Received: 29 December 2009 Accepted: 22 April 2010

Published: 22 April 2010

References

1. Jacq C, Miller JR, Brownlee GG: A pseudogene structure in 5S DNA of Xenopus laevis. Cell 1977, 12:109-120.

2. Mighell AJ, Smith NR, Robinson PA, Markham AF: Vertebrate pseudogenes. FEBS Letters 2000, 468:109-114.

3. Liu Y, Harrison P, Kunin V, Gerstein M: Comprehensive analysis of pseudogenes in prokaryotes: widespread gene decay and failure of putative horizontally transferred genes. Genome Biology 2004, 5:R64.

4. Harrison PM, Gerstein M: Studying genomes through the aeons: protein families, pseudogenes and proteome evolution. J Mol Biol 2002, 318:1155-1174.

5. Dasilva C, Hadji H, Ozouf-Costaz C, Nicaud S, Jaillon O, Weissenbach J, Crollius HR: Remarkable compartmentalization of transposable elements and pseudogenes in the heterochromatin of the Tetraodon nigroviridis genome. Proc Natl Acad Sci USA 2002, 99:13636-13641.

6. Harrison P, Kumar A, Lan N, Echols N, Snyder M, Gerstein M: A small reservoir of disabled ORFs in the yeast genome and its implications for the dynamics of proteome evolution. J Mol Biol 2002, 316:409-419.

7. Lafontaine I, Fischer G, Talla E, Dujon B: Gene relics in the genome of the yeast Saccharomyces cerevisiae. Gene 2004, 335:1-17.

8. Andersson SGE, Kurland CG: Reductive evolution of resident genomes. Trends in Microbiology 1998, 6:263-268. 
9. Andersson JO, Andersson SGE: Pseudogenes, Junk DNA, and the Dynamics of Rickettsia Genomes. Mol Biol Evol 2001, 18:829-839.

10. Cole ST, Eiglmeier K, Parkhill J, James KD, Thomson NR, Wheeler PR, Honore N, Garnier T, Churcher C, Harris D, et al:: Massive gene decay in the leprosy bacillus. Nature 2001, 409:1007-1011.

11. Gomez-Valero L, Rocha EPC, Latorre A, Silva FJ: Reconstructing the ancestor of Mycobacterium leprae: The dynamics of gene loss and genome reduction. Genome Research 2007, 17:1178-1185.

12. Lynch M, Conery JS: The evolutionary fate and consequences of duplicate genes. Science 2000, 290:1151-1155.

13. Prince VE, Pickett FB: Splitting pairs: the diverging fates of duplicated genes. Nat Rev Genet 2002, 3:827-837.

14. Vanin EF: Processed Pseudogenes: Characteristics and Evolution. Annual Review of Genetics 1985, 19:253-272.

15. Ohshima K, Hattori M, Yada T, Gojobori T, Sakaki Y, Okada N: Wholegenome screening indicates a possible burst of formation of processed pseudogenes and Alu repeats by particular L1 subfamilies in ancestral primates. Genome Biology 2003, 4:R74

16. Fischer G, Neuveglise C, Durrens P, Gaillardin C, Dujon B: Evolution of gene order in the genomes of two related yeast species. Genome Research 2001, 11:2009-2019.

17. Gesteland RF, Atkins JF: Recoding: Dynamic Reprogramming of Translation. Annual Review of Biochemistry 1996, 65:741-768.

18. Namy O, Rousset J-P, Napthine S, Brierley I: Reprogrammed Genetic Decoding in Cellular Gene Expression. Molecular Cell 2004, 13:157-168.

19. Balakirev ES, Ayala FJ: PSEUDOGENES: Are They "Junk" or Functional DNA. Annual Review of Genetics 2003, 37:123-151.

20. Duret L, Chureau C, Samain S, Weissenbach J, Avner P: The Xist RNA Gene Evolved in Eutherians by Pseudogenization of a Protein-Coding Gene. Science 2006, 312:1653-1655.

21. Rooney A, Ward T: Evolution of a large ribosomal RNA multigene family in filamentous fungi: birth and death of a concerted evolution paradigm. Proc Natl Acad Sci USA 2005, 102:5084-5089.

22. Fedorova ND, Khaldi N, Joardar VS, Maiti R, Amedeo P, Anderson MJ, Crabtree J, Silva JC, Badger JH, Albarraq A, et al.: Genomic Islands in the Pathogenic Filamentous Fungus Aspergillus fumigatus. PLoS Genet 2008, 4:e1000046.

23. Gaur M, Puri N, Manoharlal R, Rai V, Mukhopadhayay G, Choudhury D, Prasad R: MFS transportome of the human pathogenic yeast Candida albicans. BMC Genomics 2008, 9:579.

24. Hittinger CT, Rokas A, Carroll SB: Parallel inactivation of multiple GAL pathway genes and ecological diversification in yeasts. Proc Natl Acad SciUSA 2004, 101:14144-14149.

25. Dujon B, Sherman D, Fischer G, Durrens P, Casaregola S, Lafontaine I, de Montigny J, Marck C, Neuveglise C, Talla E, et al.: Genome evolution in yeasts. Nature 2004, 430:35-44.

26. Souciet J, Dujon B, Gaillardin C, Johnston M, Baret P, Cliften P, Sherman D, Weissenbach J, Westhof E, Wincker $P$, Jubin C: Comparative genomics of protoploid Saccharomycetaceae. Genome Research 2009, 19:1696-1709.

27. Sherman D, Martin T, Nikolski M, Cayla C, Souciet J, Durrens P, Consortium G: Génolevures: protein families and synteny among complete hemiascomycetous yeast proteomes and genomes. Nucleic Acids Research 2009, 37:D550-D554.

28. Pearson WR: Comparison of DNA sequences with protein sequences. Genomics 1997, 46:24-36.

29. Pearson WR: Empirical statistical estimates for sequence similarity searches. Journal of Molecular Biology 1998, 276:71-84.

30. Finn RD, Mistry J, Tate J, Coggill P, Heger A, Pollington JE, Gavin OL, Gunasekaran P, Ceric G, Forslund K, et al:: The Pfam protein families database. Nucl Acids Res 2010, 38:D211-222.

31. Eddy SR: Profile hidden Markov models. Bioinformatics 1998 14:755-763.

32. Doniger SW, Kim HS, Swain D, Corcuera D, Williams M, Yang S-P, Fay JC: A Catalog of Neutral and Deleterious Polymorphism in Yeast. PLoS Genet 2008, 4:e1000183.

33. Birney E, Clamp M, Durbin R: GeneWise and Genomewise. Genome Research 2004, 14:988-995

34. Durrens $P$, Sherman D: A systematic nomenclature of chromosomal elements for hemiascomycete yeasts. Yeast 2005, 22:337-342.

35. Edgar RC: MUSCLE: multiple sequence alignment with high accuracy and high throughput. Nucl Acids Res 2004, 32:1792-1797.
36. R Development Core Team: $R$ : A language and environment for statistical computing Vienna, Austria; 2005. ISBN 3-900051-07-0

37. Sacerdot C, Casaregola I, Lafontaine I, Tekaia F, Dujon B, OzierKalogeropoulos O: Promiscuous DNA in the nuclear genomes of hemiascomycetous yeasts. FEMS Yeast Research 2008, 8:846-857.

38. Farabaugh PJ, Kramer E, Vallabhaneni H, Raman A: Evolution of +1 programmed frameshifting signals and frameshift-regulating tRNAs in the order Saccharomycetales. J Mol Evol 2006, 63:545-561.

39. Richard G-F, Kerrest A, Lafontaine I, Dujon B: Comparative Genomics of Hemiascomycete Yeasts: Genes Involved in DNA Replication, Repair and Recombination. Mol Biol Evol 2005, 22:1011-1023.

40. Dujon B: Yeasts illustrate the molecular mechanisms of eukaryotic genome evolution. Trends in Genetics 2006, 22:375-387.

41. Fairhead C, Dujon B: Structure of Kluyveromyces lactis subtelomeres: duplications and gene content. FEMS Yeast Research 2006, 6:428-441.

42. Mondoux M, Zakian V: Telomere position effect: silencing near the end. In Telomeres 2nd edition. Edited by: de Lange T, Lundblad V, Blackburn E. Cold Spring Harbor, New York: Cold Spring Harbor Laboratory Press; 2006:261-316

43. Derr LK, Strathern JN, Garfinkel DJ: RNA-mediated recombination in S. cerevisiae. Cell 1991, 67:355-364.

44. Wolfe KH, Shields DC: Molecular evidence for an ancient duplication of the entire yeast genome. Nature 1997, 387:708-713.

45. Byrne KP, Wolfe KH: The Yeast Gene Order Browser: Combining curated homology and syntenic context reveals gene fate in polyploid species. Genome Research 2005, 15:1456-1461.

46. Balasubramanian S, Zheng D, Liu Y, Fang G, Frankish A, Carriero N, Robilotto R, Cayting P, Gerstein M: Comparative analysis of processed ribosomal protein pseudogenes in four mammalian genomes. Genome Biology 2009, 10:R2.

47. Svensson O, Arvestad L, Lagergren J: Genome-Wide Survey for Biologically Functional Pseudogenes. PLoS Computational Biology 2006, 2:e46.

48. Payen C, Koszul R, Dujon B, Fischer G: Segmental duplications arise from pol32 dependent repair of broken forks through two alternative replication-based mechanisms. PLoS Genetics 2008, 4:e1000175.

49. Gordon JL, Byrne KP, Wolfe KH: Additions, Losses, and Rearrangements on the Evolutionary Route from a Reconstructed Ancestor to the Modern Saccharomyces cerevisiae Genome. PLoS Genet 2009, 5:e1000485.

50. Lynch M, Sung W, Morris K, Coffey N, Landry C, Dopman E, Dickinson W, Okamoto K, Kulkarni S, Hartl D, Thomas W: A genome-wide view of the spectrum of spontaneous mutations in yeast. Proc Natl Acad Sci USA 2008.

51. Zheng D, Frankish A, Baertsch R, Kapranov P, Reymond A, Choo S, Lu Y, Denoeud F, Antonarakis S, Snyder M, et al:: Pseudogenes in the ENCODE regions: consensus annotation, analysis of transcription, and evolution. Genome Research 2007, 17:839-851

52. Schacherer J, Tourrette $Y$, Souciet J-L, Potier S, de Montigny J: Recovery of a Function Involving Gene Duplication by Retroposition in Saccharomyces cerevisiae. Genome Res 2004, 14:1291-1297.

53. Maxwell P, Coombes C, Kenny A, Lawler J, Boeke J, Curcio M: Ty1 mobilizes subtelomeric $Y^{\prime}$ elements in telomerase-negative Saccharomyces cerevisiae survivors. Molecular and Cellular Biology 2004, 24:9887-9898

54. Neuveglise C, Feldmann H, Bon E, Gaillardin C, Casaregola aS: Genomic Evolution of the Long Terminal Repeat Retrotransposons in Hemiascomycetous Yeasts. Genome Res 2002, 12:930-943.

55. Koonin E, Mushegian A, Bork P: Non-orthologous gene displacement. Trends in Genetics 1996, 12:334-336.

56. Pena-Castillo L, Hughes TR: Why Are There Still Over 1000 Uncharacterized Yeast Genes? Genetics 2007, 176:7-14.

57. Fabre E, Muller $H$, Therizols $P$, Lafontaine I, Dujon B, Fairhead C: Comparative Genomics in Hemiascomycete Yeasts: Evolution of Sex, Silencing, and Subtelomeres. Mol Biol Evol 2003, 33:29-45.

58. Barry JD, Ginger ML, Burton P, McCulloch R: Why are parasite contingency genes often associated with telomeres? Int J Parasitol 2003, 33:29-45.

59. Teytelman L, Eisen MB, Rine J: Silent but Not Static: Accelerated BasePair Substitution in Silenced Chromatin of Budding Yeasts. PLoS Genet 2008, 4:e1000247. 
60. Pu S, Wong J, Turner B, Cho E, Wodak S: Up-to-date catalogues of yeast protein complexes. Nucleic Acids Research 2009, 37:825-831.

61. Costanzo M, Baryshnikova A, Bellay J, Kim Y, Spear ED, Sevier CS, Ding H, Koh JLY, Toufighi K, Mostafavi S, et al:: The Genetic Landscape of a Cell. Science 2010, 327:425-431.

62. Lior D, Huber W, Granovskaia M, Toedling J, Palm CJ, Bofkin L, Jones T, Davis RW, Steinmetz LM: A high-resolution map of transcription in the yeast genome. PNAS 2006, 103:5320-5325.

63. Neil H, Malabat C, d'Aubenton-Carafa Y, Xu Z, Steinmetz LM, Jacquier A: Widespread bidirectional promoters are the major source of cryptic transcripts in yeast. Nature 2009, 457:1038-1042.

64. Nagalakshmi U, Wang Z, Waern K, Shou C, Raha D, Gerstein M, Snyder M: The Transcriptional Landscape of the Yeast Genome Defined by RNA Sequencing. Science 2008, 320:1344-1349.

65. He F, Li X, Spatrick P, Casillo R, Dong S, Jacobson A: Genome-Wide Analysis of mRNAs Regulated by the Nonsense-Mediated and 5' to 3' mRNA Decay Pathways in Yeast. Molecular Cell 2003, 12:1439-1452.

66. Namy O, Duchateau-Nguyen G, Hatin I, Hermann-Le Denmat S, Termier M, Rousset J: Identification of stop codon readthrough genes in Saccharomyces cerevisiae. Nucleic Acids Research 2003, 31:2289-2296.

67. Chambers JM, Cleveland WS, Tukey PA: Graphical Methods for Data Analysis Duxbury Press; 1983

68. Kurtzman CP: Phylogenetic circumscription of Saccharomyces, Kluyveromyces and other members of the Saccharomycetaceae, and the proposal of the new genera Lachancea, Nakaseomyces, Naumovia, Vanderwaltozyma and Zygotorulaspora. FEMS Yeast Research 2003, 4:233-245.

69. Guindon S, Gascuel O: A simple, fast, and accurate algorithm to estimate large phylogenies by maximum likelihhod. Syst Biol 2003, 52:696-704

70. Wolfe KH: Yesterday's polyploids and the mystery of diploidization. Nat Rev Genet 2001, 2:333-341.

doi: 10.1186/1471-2164-11-260

Cite this article as: Lafontaine and Dujon, Origin and fate of pseudogenes in Hemiascomycetes: a comparative analysis BMC Genomics 2010, 11:260

Submit your next manuscript to BioMed Centra and take full advantage of:

- Convenient online submission

- Thorough peer review

- No space constraints or color figure charges

- Immediate publication on acceptance

- Inclusion in PubMed, CAS, Scopus and Google Scholar

- Research which is freely available for redistribution

Submit your manuscript at www.biomedcentral.com/submit
C Biomed Central 